\section{Efficacy of Early Treatment with Onabotulinum Toxin A in Post-Stroke Spasticity}

\section{Aránzazu Vázquez*, Juan Antonio Caballero, María Pia Spottorno, Elsa García, Laura García and Miguel Ángel Hernández}

Department of Physical Medicine and Rehabilitation, Princess University Hospital, Madrid, Spain

\begin{abstract}
Background

To compare the effect of early versus delayed administration of Onabotulinum Toxin Type A (OnabotA) injections in patients with post-stroke spasticity in the Upper (UL) and/or Lower Limbs (LL).

Methods

A retrospective study, from 2014 to 2017 , was conducted on consecutive patients with moderate to severe stroke according to the National institute of Health Stroke Scale (NIHSS) [NIHSS score 14 to 25]. All patients received individualized treatment with OnabotA (Allergan Inc., Irvine, CA, USA) at baseline in the affected limbs. Study patients were divided in two groups; Group I: Administration of Onabot A within the first 6 months after stroke and Group II: Administration of Onabot A later than 6 months after stroke. The primary end-point was the changes in muscle tone, assessed by means of the Modified Ashworth Scale (MAS), at the end of the 1-year treatment period.

Results

Of 162 screened patients, 32 met the inclusion/exclusion criteria and were included in the study. In the LL, the MAS score reduction was significantly greater $(p=0.0452)$ in those patients who received OnabotA treatment at early stages. Similarly, the Onabot A dose received throughout the study was significantly lower in patients receiving toxin treatment early in the process $(p=0.0003)$. For each
\end{abstract}

*Corresponding author: Aránzazu Vázquez, Department of Physical Medicine and Rehabilitation, Princess University Hospital, Madrid, Spain, Tel: +34 915202367; E-mail: vazquezdoce@hotmail.com

Citation: Vázquez A, Caballero JA, Spottorno MP, García E, García L, et al. (2018) Efficacy of Early Treatment with Onabotulinum Toxin A in Post-Stroke Spasticity. J Phys Med Rehabil Disabil 4: 025.

Received: January 17, 2018; Accepted: March 09, 2018; Published: March 22, 2018

Copyright: (C 2018 Vázquez A, et al., This is an open-access article distributed under the terms of the Creative Commons Attribution License, which permits unrestricted use, distribution, and reproduction in any medium, provided the original author and source are credited. day that treatment with OnabotA was delayed, MAS score was 0.003 units $(E E=0.001)$ greater.

\section{Conclusion}

The results of this study suggested that early treatment with Onabot $\mathrm{A}$ administered in post-stroke spasticity patients was associated with a better evolution of the process.

Keywords: Botulinumtoxin A; Early treatment; Modifies Ashworth scale; Postroke pain; Spasticity; Stroke

\section{Introduction}

Post-Stroke Spasticity (PSS) has been described as a relevant clinical problem in stroke survivors, as it can impair manual dexterity, mobility and balance, with a negative impact on independence [1]. As a result, spasticity has been hypothesized to have a significant negative impact on the health-related quality of life of stroke survivors [2].

Patients who do not recover some functional activity during the early stages (4 to 6 weeks after the stroke) have greater risk of developing long-term musculoskeletal complications such as contractures and pain [3]. Therefore, early recognition of spasticity and identification of predictors to recognize those stroke patients who are at risk of developing spasticity, are necessary to yield earlier treatment of PSS and possibly better outcomes $[4,5]$.

The incidence of spasticity depends on its etiology. PSS is a common sequela with an incidence ranging from $17 \%$ to $43 \%$ [6,7], which is greater in younger patients [8] and the upper limb [6,9]. In an observational, multicenter study, conducted in patients with spasticity in Spain, it was shown that $63 \%$ of patients had PSS [10].

Scientific evidence shows that approximately $19 \%$ of patients present spasticity within 3 months of having a stroke [11] and this proportion increases to $38 \%$ at month 12 [12]. However, these percentages may vary depending on etiology, comorbidity, and available rehabilitation treatments.

The management of spasticity is a complex process that should be ideally is approached by a multidisciplinary team. Currently, spasticity is frequently managed through a combination of therapeutic modalities, including physiotherapy, oral medications (baclofen, tizanidine, dantrolene or benzodiazepines), chemoneurolysis procedures (phenol or ethyl alcohol) and intrathecal baclofen pump $[13,14]$. In addition, administration of BoNTA has been identified as an effective and safe treatment for the management of spasticity in routine clinical practice [15-21].

So far treatment of spasticity is currently delayed until patient develops signs of these complications. Although there is new evidence recommending to start with the treatment within the three months of the stroke onset $[22,23]$, in a classical way, treatment of spasticity was usually delayed until secondary complications are established, approximately 6 months after the stroke [20]. Moreover, it is possible that deterioration in the musculoskeletal impairments before the initiation of spasticity treatment may be detrimental to the patient as these 
musculoskeletal changes may have become too chronic and impossible to fully eradicate [24].

The aim of this study is to compare the effect of early versus delayed administration of Onabotulinum Toxin Type A (OnabotA) injections in patients with post-stroke spasticity in the Upper (UL) and/ or Lower Limbs (LL).

\section{Methods}

A Retrospective study, from 2014 to 2017 , was conducted on consecutive patients with moderate to severe stroke according to the National Institute of Health Stroke Scale (NIHSS) [25] [NIHSS score 14 to 25]. The potential study population was all the patients seen at the outpatient service of the Physical Medicine and Rehabilitation Department of the Princess University Hospital, between 2010 and 2015, and met the inclusion criteria.

The study protocol was approved by the ethics committee of the Princess University (CIEC 2830, July 7, 2016) Hospital and conducted in accordance with the Declaration of Helsinki. The need for informed consent was waived in view of the retrospective and observational nature of the study.

Eligible patients were aged 18 years or older with evidence of upper or lower limb spasticity established by a specialist in Physical Medicine and Rehabilitation [UL or LL spasticity was defined by a score in the Modified Ashworth Scale (MAS) [26,27] equal or greater than 1]; Have a NIHSS score post-fibrinolysis ranging from 14 to 25 . Score ranging from 14 to 25 ; to carry out the PSS rehabilitation protocol of the Princess University Hospital; and have a minimum follow-up period of 12 months under BoNTA treatment.

All the patients included had to meet the currently established criteria as early predictors of spasticity [28] and do not present previous functional alterations with a premorbid BARTHEL basal index of 100 .

Those patients with concomitant diseases that, in the opinion of the investigator, could confuse or difficult the proper assessment of spasticity; appearance of a new central vascular event during the follow-up; who were participating in a clinical trial; or have any condition considered to be a contraindication to BoNTA (allergic reaction, diseases of the neuromuscular junction, peripheral neuropathic diseases, etc.) were excluded from the analysis.

At baseline, patients may be receiving conventional anti-spastic therapy, including oral anti-spastic medications, physiotherapy and occupational therapy at the discretion of the specialist.

All patients received individualized treatment with OnabotA (Allergan Inc., Irvine, CA, USA) at baseline in the affected limbs, as determined by clinical factors, spasticity patterns, specialist preference, and treatment goals $[16,22,29]$.

OnabotA injections (one or two per injected muscle, according to patient characteristics), contained a preparation of 100 units of diluted with $1 \mathrm{cc} 0.9 \%$ saline and were injected by ultrasound guidance.

Study patients were divided into two groups, depending on the time of starting the OnabotA treatment; Group I: Administration of OnabotA within the first 6 months after stroke and Group II: Administration of OnabotA later than 6 months after stroke.
The primary end-point was the change in muscle tone, assessed by means of the modified Ashworth scale reduction, at the end of the 1 -year treatment period.

The MAS scores were assessed for five muscle groups in the UL (wrist flexors, elbow flexors, elbow extensors, shoulder abductors and shoulder adductors) and six in the LL (hip flexors, hip adductors, knee extensors, knee flexors, plantar flexors and ankle-dorsal flexors). The M AS is a 6-point scale [0 (no increase in muscle tone) to 4 (affected part (s) rigid in flexion or extension)] that measures resistance to passive movement across a joint $[26,27]$. Mean MAS score in the UL or LL was calculated as the average of MAS scores on muscles of the UL or LL on the affected body side, defined as the side with the highest average MAS score at baseline.

Secondary endpoints included pain reduction assessed by Visual Analogue Scale (VAS) [30]; gait capability [assessed as autonomous walking capacity with technical supports (cane or orthosis) or without autonomous walking capacity]; need of concomitant medication for pain; number of infiltrated muscles (as a subrogate of less spasticity, regardless of spastic pattern); and Barthel's index at the end of the study follow-up [31].

\section{Statistical Analysis}

A standard statistical analysis was performed using MedCalc 17.5.3 (MedCalc Software bvba, Ostend, Belgium).

Before the study, it was determined that a sample of at least 15 patients per group was required to detect a difference of 1 unit in mean MAS score change at a significance level of 0.05 , with a power of 0.80 and assuming a standard deviation of 0.9 . A follow-up loss rate of the $15 \%$ has been estimated.

Data are expressed as number (percentage), mean [Standard Deviation (SD)], mean [95\% confidence Interval $(95 \% \mathrm{CI})]$, or median $(95 \% \mathrm{CI})$ as appropriate. We examined the distribution of continuous variables with a D'Agostino-Pearson test.

If data were normally distributed, one-way Analysis of Variance (ANOVA) was used to compare means between treatment groups for quantitative variables at baseline. Two-way ANOVA was used to evaluate the effect of treatments and the time factor. When a normal distribution was not expected the Kruskal-Wallis test was used.

Categorical variables were compared using a Chi-square test and a Fisher's exact test, as needed.

To quantify the relationship between the OnabotA doses and the changes in MAS and pain, as dependent variables, and time to administration of OnabotA, as independent variable, a linear regression analysis was performed. For linear regression analysis, the Pearson correlation coefficient (r) was used.

A probability value of $\mathrm{p}, 0.05$ was considered indicative of statistical significance.

\section{Results}

Of 162 screened patients, 32 met the inclusion/exclusion criteria. Their main characteristics are shown in table 1.

Regarding the severity of the process (according to the post-fibrinolysis NIHSS score), in group I, 2 (12.5\%) patients were classified 
Citation: Vázquez A, Caballero JA, Spottorno MP, García E, García L, et al. (2018) Efficacy of Early Treatment with Onabotulinum Toxin A in Post-Stroke Spasticity. J Phys Med Rehabil Disabil 4: 025.

- Page 3 of $7 \bullet$

as moderate and $14(87.5 \%)$ as severe; compared to $1(6.3 \%)$ classified as moderate and $15(93.7 \%)$ classified as severe in group II.

\begin{tabular}{|c|c|c|c|}
\hline & Group I & Group II & \\
\hline & $(n=16)$ & $(n=16)$ & \\
\hline \multicolumn{3}{|l|}{ Age, years } & \multirow{3}{*}{$0.3627^{*}$} \\
\hline Mean (SD) & $63.7(13.2)$ & $59.6(12.0)$ & \\
\hline $95 \% \mathrm{CI}$ & 56.6 a 70.7 & 53.2 a 66.0 & \\
\hline \multicolumn{3}{|l|}{ Sex, n (\%) } & \multirow{3}{*}{$1.000^{* *}$} \\
\hline Man & $9(56.3)$ & $8(50.0)$ & \\
\hline Woman & $7(43.7)$ & $8(50.0)$ & \\
\hline \multicolumn{3}{|l|}{ NIHSS } & \multirow{3}{*}{$0.0153 *$} \\
\hline Mean (SD) & $18.6(3.7)$ & $16.0(1.7)$ & \\
\hline $95 \% \mathrm{CI}$ & 16.7 a 20.6 & 15.1 a 16.9 & \\
\hline \multicolumn{3}{|l|}{ Barthel index } & \multirow{3}{*}{$1.000^{*}$} \\
\hline Mean (SD) & $98.8(3.4)$ & $98.8(5.0)$ & \\
\hline $95 \% \mathrm{CI}$ & 96.9 a 100.6 & 96.1 a 101.4 & \\
\hline \multicolumn{3}{|l|}{ Location, n (\%) } & \multirow{3}{*}{$0.7231^{* *}$} \\
\hline Right & $7(43.7)$ & $6(37.5)$ & \\
\hline Left & $9(66.3)$ & $10(62.5)$ & \\
\hline \multicolumn{3}{|l|}{ Diagnostic, n (\%) } & \multirow{3}{*}{$0.8145^{* *}$} \\
\hline Ischemic stroke & $10(62.5)$ & $9(66.3)$ & \\
\hline Hemorrhagic stroke & $6(37.5)$ & $7(43.7)$ & \\
\hline \multicolumn{3}{|l|}{ MAS UL, n (\%) } & \multirow{7}{*}{$0.0281 * *$} \\
\hline 1 & $1(6.3)$ & $0(0.0)$ & \\
\hline 1.5 & $0(0.0)$ & $0(0.0)$ & \\
\hline 2 & $8(50.0)$ & $2(12.5)$ & \\
\hline 2.5 & $1(6.3)$ & $0(0.0)$ & \\
\hline 3 & $5(31.1)$ & $14(87.5)$ & \\
\hline 4 & $1(6.3)$ & $0(0.0)$ & \\
\hline \multicolumn{3}{|l|}{ MAS LL, n (\%) } & \multirow{7}{*}{$0.2392 * *$} \\
\hline 1 & $4(25.0)$ & $3(18.7)$ & \\
\hline 1.5 & $1(6.3)$ & $0(0.0)$ & \\
\hline 2 & $6(37.5)$ & $5(31.1)$ & \\
\hline 2.5 & $0(0.0)$ & $0(0.0)$ & \\
\hline 3 & $3(18.7)$ & $8(50.0)$ & \\
\hline 4 & $2(12.5)$ & $0(0.0)$ & \\
\hline \multicolumn{3}{|l|}{ Aphasia, n (\%) } & \multirow{3}{*}{$0.4830^{* *}$} \\
\hline Yes & $8(50.0)$ & $6(37.5)$ & \\
\hline No & $8(50.0)$ & $10(62.5)$ & \\
\hline \multicolumn{3}{|l|}{ Dysphagia, n (\%) } & \multirow{3}{*}{$0.4864 * *$} \\
\hline Yes & $7(43.7)$ & $9(66.3)$ & \\
\hline No & $9(66.3)$ & $7(43.7)$ & \\
\hline \multicolumn{3}{|l|}{ Gait, n (\%) } & \multirow{3}{*}{$0.2956^{* *}$} \\
\hline Autonomous walking & $0(0.0)$ & $0(0.0)$ & \\
\hline Walking with technical supports ${ }^{1}$ & $6(37.5)$ & $9(56.3)$ & \\
\hline without autonomous walking & $10(62.5)$ & $7(43.7)$ & \\
\hline \multicolumn{3}{|l|}{ Spasticity treatment, n (\%) } & \\
\hline None & $4(25.0)$ & $1(6.3)$ & \\
\hline Baclofen & $7(43.7)$ & $0(0.0)$ & $0.0012 * *$ \\
\hline Gabapentin & $4(25.0)$ & $2(12.5)$ & \\
\hline Baclofen + gabapentin & $0(0.0)$ & $4(25.0)$ & \\
\hline
\end{tabular}

\begin{tabular}{|c|c|c|c|}
\hline Baclofen + analgesia & $1(6.3)$ & $6(37.5)$ & \\
\hline Analgesia + neuromodulators & $0(0.0)$ & $3(18.7)$ & \\
\hline \multicolumn{4}{|c|}{$\begin{array}{l}\text { Abbreviations: } \\
\mathrm{n}=\text { number; } \mathrm{SD}=\text { Standard Deviation; CI=Confidence Interval; NIHSS=National Insti- } \\
\text { tute of Health Stroke Scale MAS=Modified Ashworth Scale } \\
{ }^{1} \text { : Cane or orthosis } \\
\text { *One-way ANOVA } \\
* * \text { Chi-square Test }\end{array}$} \\
\hline
\end{tabular}

The mean time $(95 \% \mathrm{CI})$ from stroke to the start of OnabotA treatment was 90.4 (68.4 to 112.5) and 272.4 (225.1 to 319.7) days in groups I and II, respectively, $\mathrm{p}<0.0001$.

The mean dose (SD) of OnabotA administered at baseline was 337.5 (71.9) $\mathrm{U}$ and 393.8 (44.3) $\mathrm{U}$ in groups I and II, respectively, $\mathrm{p}=0.012$.

At baseline, the mean (SD) number of infiltrated muscles in the UL were significantly greater in group II [6.9 (1.8); 95\% CI: 5.8 to 7.7] than in group I [3.3 (1.6); $95 \%$ CI: 2.4 to 4.2], $<<0.001$. However, there was no significant difference between the mean number of LL infiltrated muscles between groups I [2.8 (1.9); 95\% CI: 1.7 to 3.8] and II [4.2 (2.1); 95\% CI: 3.1 to 5.3], $\mathrm{p}=0.0523$.

The pain degree at baseline was significantly greater in group II [7.1 (2.1); 95\% CI: 5.9 to 8.2] than in group I [5.3 (2.5); 95\% CI: 3.9 to 6.7$], \mathrm{p}=0.0419$.

During the 12-month minimum follow-up of the study, the mean (SD) number of infiltrations was similar in both groups: 3.6 (1.0) versus $4.0(0.0)$ in groups I and II, respectively, $\mathrm{p}=0.100$.

The table 2 summarizes the results of the main study results at the end of the study follow-up period.

There was an overall improvement in mean MAS in the upper limb in both groups (Figure 1), with a greater improvement observed in group I than in the group II (mean change -0.72 versus -0.38 , respectively) (Table 3 ).

The differences observed between the two study groups in UL and LL MAS, OnabotA dose, and number of UL and/or LL treated muscles after one year of treatment are shown in table 3 .

In the LL, the MAS score reduction was significantly greater $(p=0.0452)$ in those patients who received OnabotA treatment at early stages.

The mean (SD) $(95 \% \mathrm{CI})$ total dose of OnabotA administered throughout the study follow-up was significantly greater in group II [843.8 (81.4) U, 95\% CI 800.4 to $887.1 \mathrm{U}$ ] than in group I [593.8 (163.2) U, $95 \%$ CI 506.8 to $680.7 \mathrm{U}], \mathrm{p}<0.0001$.

Similarly, the OnabotA dose received throughout the study was significantly lower in patients receiving toxin treatment early in the process $(\mathrm{p}=0.0003)($ Table 3$)$.

The number of patients requiring anti-spastic treatment significantly decreased after OnabotA treatment $[12(37.5 \%)]$ as compared to baseline [5 (12.5\%)], $\mathrm{p}=0.0491$. 
Citation: Vázquez A, Caballero JA, Spottorno MP, García E, García L, et al. (2018) Efficacy of Early Treatment with Onabotulinum Toxin A in Post-Stroke Spasticity. J Phys Med Rehabil Disabil 4: 025.

\begin{tabular}{|c|c|c|c|}
\hline & Group I & Group II & \multirow{2}{*}{$\mathbf{P}$} \\
\hline & $(n=16)$ & $(n=16)$ & \\
\hline \multicolumn{3}{|l|}{ MAS UL, $\mathbf{n}(\%)$} & \multirow{7}{*}{$0.0012 * *$} \\
\hline 1 & $5(31.3)$ & $0(0.0)$ & \\
\hline 1.5 & $0(0.0)$ & $0(0.0)$ & \\
\hline 2 & $11(68.7)$ & $8(50.0)$ & \\
\hline 2.5 & $0(0.0)$ & $8(50.0)$ & \\
\hline 3 & $0(0.0)$ & $0(87.5)$ & \\
\hline 4 & $0(0.0)$ & $0(0.0)$ & \\
\hline \multicolumn{3}{|l|}{ MAS LL, n (\%) } & \multirow{7}{*}{$0.0206 * *$} \\
\hline 1 & $10(62.5)$ & $3(18.8)$ & \\
\hline 1.5 & $0(0.0)$ & $0(0.0)$ & \\
\hline 2 & $6(37.5)$ & $10(62.5)$ & \\
\hline 2.5 & $0(0.0)$ & $0(0.0)$ & \\
\hline 3 & $0(0.0)$ & $3(18.8)$ & \\
\hline 4 & $0(0.0)$ & $0(0.0)$ & \\
\hline \multicolumn{3}{|l|}{ Gait, n (\%) } & \multirow{4}{*}{$0.0174 * *$} \\
\hline Autonomous walking & $8(50.0)$ & $2(12.5)$ & \\
\hline Walking with technical supports ${ }^{1}$ & $7(43.8)$ & $7(43.7)$ & \\
\hline Without autonomous walking & $1(6.2)$ & $7(43.7)$ & \\
\hline \multicolumn{3}{|l|}{ Spasticity treatment, n (\%) } & \multirow{8}{*}{$0.0322 * *$} \\
\hline None & $7(43.7)$ & $5(31.3)$ & \\
\hline Baclofen & $8(50.0)$ & $1(6.2)$ & \\
\hline Gabapentin & $1(6.2)$ & $3(18.8)$ & \\
\hline Analgesia & $0(0.0)$ & $3(18.8)$ & \\
\hline Baclofen + gabapentin & $0(0.0)$ & $1(6.2)$ & \\
\hline Baclofen + analgesia & $0(0.0)$ & $2(12.5)$ & \\
\hline Analgesia + neuromodulators & $0(0.0)$ & $1(6.2)$ & \\
\hline \multicolumn{3}{|l|}{ NIM UL } & \multirow{3}{*}{$<0.001 *$} \\
\hline Mean (SD) & $2.9(1.6)$ & $6.4(1.5)$ & \\
\hline $95 \% \mathrm{CI}$ & 2.0 to 3.7 & 5.6 to 7.2 & \\
\hline \multicolumn{3}{|l|}{ NIM LL } & \multirow{3}{*}{$0.017 *$} \\
\hline Mean (SD) & $1.9(1.5)$ & $3.2(1.4)$ & \\
\hline $95 \% \mathrm{CI}$ & 1.1 to 2.7 & 2.4 to 4.0 & \\
\hline \multicolumn{3}{|l|}{ Barthel index } & \multirow{3}{*}{$0.632 *$} \\
\hline Mean (SD) & $78.4(22.6)$ & $70.3(25.6)$ & \\
\hline $95 \% \mathrm{CI}$ & 66.4 to 90.5 & 56.7 to 83.9 & \\
\hline
\end{tabular}

Table 2: Overview of the results of as worth scale scores in Upper (UL) and Lower (LL) Limbs; gait capability; need of concomitant medication, number of infiltrated muscles; and Barthel's index at the end of the study follow-up period.

Abbreviations:

$\mathrm{n}=$ number; $\mathrm{SD}=$ Standard Deviation; $\mathrm{CI}=$ Confidence Interval; MAS=Modified Ashworth Scale; NIM=Number of Infiltrated Muscles

${ }^{1}$ : Cane or Orthosis

* One-way ANOVA

**Chi square Test

The figure 2 shows the relationship between the time elapsed between the stroke and the beginning of the OnabotA treatment and the dose of OnabotA administered at month 12 (A) and the value of LL MAS at the end of the follow-up (B).

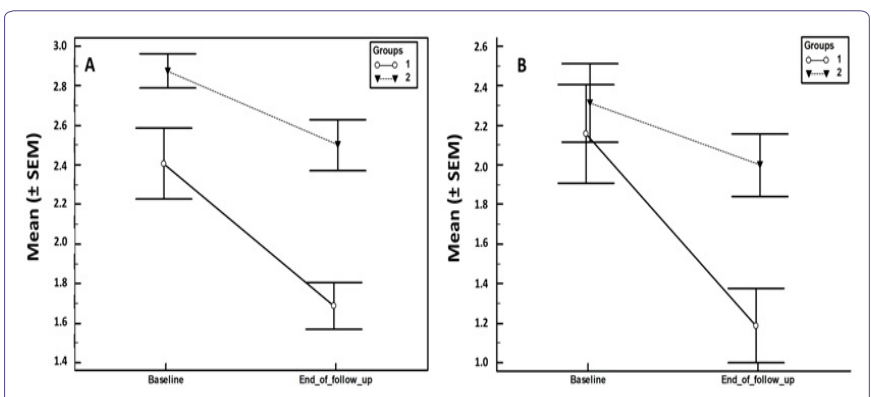

Figure 1: Mean \pm Standard Error of the Mean (SEM) Modified Ashworth scale (MAS) score in the upper (A) and lower (B). Limbs at baseline and the end of follow-up period in the study groups.

For each day that the start of the OnabotA treatment was delayed, the total dose administered throughout the study increases by $0.82 \mathrm{U}$ (standard error $[\mathrm{EE}]=0.24$ ). Additionally, for each day that treatment with OnabotA was delayed, the MAS score was 0.003 units $(\mathrm{EE}=0.001)$ greater.

The table 4 shows the correlation between the time elapsed between stroke onset and the beginning of OnabotA treatment and different study variables.

The time elapsed between stroke onset and the start of OnabotA treatment correlates significantly with UL MAS score $(\mathrm{r}=0.49$, $\mathrm{p}=0.0041)$; LL MAS score $(\mathrm{r}=0.46, \mathrm{p}=0.0077)$; pain $(\mathrm{r}=0.56$, $\mathrm{p}=0.0009)$; number of UL infiltrated muscles $(\mathrm{r}=0.58, \mathrm{p}=0.0006)$; and the total dose of OnabotA $(\mathrm{r}=0.52, \mathrm{p}=0.0021)$ (Table 4).

\begin{tabular}{|c|c|c|c|c|c|l|}
\hline & \multicolumn{3}{|c|}{ Group I } & \multicolumn{2}{c|}{ Group II } & \multicolumn{2}{c|}{ Differences Between Groups } \\
\cline { 2 - 7 } & $\begin{array}{c}\text { Mean (95\% CI) } \\
\text { difference from baseline }\end{array}$ & $\mathbf{p}$ & $\begin{array}{c}\text { Mean (95\% CI) } \\
\text { difference from baseline }\end{array}$ & $\mathbf{p}$ & Mean (95\% CI) & \multicolumn{1}{|c|}{ p } \\
\hline MAS UL & $-0.72(-1.08$ to -0.36$)$ & 0.0008 & $-0.38(-0.64$ a -0.11$)$ & 0.0090 & $-0.34(-0.78$ a 0.09$)$ & 0.1145 \\
\hline MAS LL & $-0.97(-1.56$ a -0.38$)$ & 0.0031 & $-0.31(-0.63$ a 0.01$)$ & 0.0555 & $-0.66(-1.30$ a -0.02$)$ & 0.0452 \\
\hline OnabotA dose & $-81.3(-135.3$ a -27.2$)$ & 0.0059 & $56.3(8.7$ a 103.8$)$ & 0.0235 & $137.5(68.5$ a 206.5$)$ & 0.0003 \\
\hline NIM UL & $-0.44(-1.47$ a 0.59$)$ & 0.3791 & $-0.31(-1.08$ a 0.46$)$ & 0.4015 & $-0.13(-1.36$ a 1.11$)$ & 0.8373 \\
\hline NIM LL & $-0.88(-1.97$ a 0.22$)$ & 0.1102 & $-1.00(-1.91$ a -0.09$)$ & 0.0338 & $0.12(-1.24$ a 1.49$)$ & 0.8533 \\
\hline Pain & $-4.3(-5.8$ a -2.8$)$ & $<0.0001$ & $-4.0(-5.3$ a -2.6$)$ & $<0.0001$ & $-0.27(-2.19$ to 1.66$)$ & 0.7790 \\
\hline
\end{tabular}

Table 3: Overview of Ashworth modified scale values in upper and lower limbs, botulinum toxin dose and number of upper and lower limb infiltrated muscles and their changes from baseline.

Abbreviations:

$\mathrm{CI}=$ Confidence Interval; MAS=Modified Ashworth Scale; UL=Upper Limb; LL=Lower Limb; OnabotA=Onabotulinumtoxin A; NIM=Number of Infiltrated Muscles 

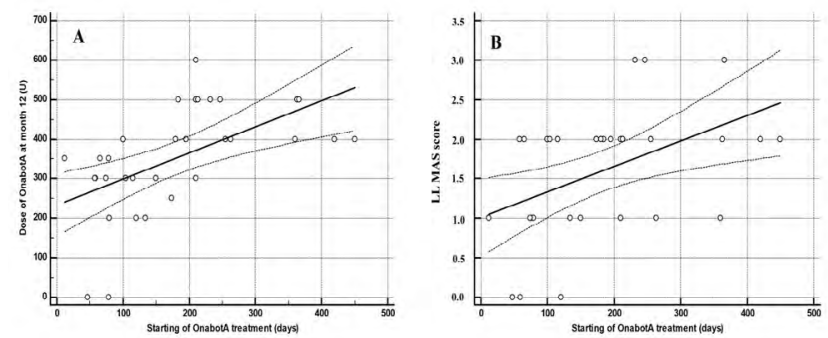

Figure 2: Evaluation of the relationship between the time Onabotulinumtoxin A (OnabotA) treatment onset and the dose of OnabotA administered at month 12 of the study and the modified Ashworth scale score. (A) There was a significant relationship between the time elapsed between stroke onset and the start of the OnabotA treatment and the total dose of Onabot $\mathrm{A}$ administered throughout the study $(\mathrm{r}=0.55$, Confidence Interval $[\mathrm{CI}] 95 \%=0.25$ to $0.76, \mathrm{p}=0.0011$ ). (B) There was a significant relationship between the time of OnabotA treatment onset and the Lower Limb (LL) modified Ashworth scale score at the end of the follow-up ( $r=0.46$; CI $95 \%=0.14$ to $0.70 ; p=0.0077$ ).

\begin{tabular}{|c|c|c|c|}
\hline & \multicolumn{4}{|c|}{$\begin{array}{c}\text { Time Elapsed Between Stroke Onset and the Start } \\
\text { of OnabotA Treatment (Days) }\end{array}$} \\
\hline & $\mathbf{R}$ & $\mathbf{9 5 \%}$ CI & p \\
\hline MAS UL & 0.49 & 0.18 to 0.72 & 0.0041 \\
\hline MAS LL & 0.46 & 0.14 to 0.70 & 0.0077 \\
\hline Barthel index & -0.09 & -0.43 to 0.26 & 0.6054 \\
\hline Pain & 0.56 & 0.26 to 0.76 & 0.0009 \\
\hline NIM UL & 0.58 & 0.28 to 0.77 & 0.0006 \\
\hline NIM LL & 0.27 & -0.09 to 0.57 & 0.1340 \\
\hline OnabotA total dose & 0.52 & 0.21 to 0.74 & 0.0021 \\
\hline
\end{tabular}

Table 4: Relationship between the time elapsed between stroke onset and the start of Onabotulinumtoxin A (OnabotA) treatment and different spasticity variables at the end of the follow-up period.

\section{Abbreviations:}

$\mathrm{CI}=$ Confidence Interval; MAS=Modified Ashworth Scale; UL=Upper Limb; LL=Lower Limb; NIM=Number of Infiltrated Muscles; OnabotA=Onabotulinumtoxin A.

At the end of the follow-up, the number of patients with autonomous walking capacity was significantly greater in the group I [ $93.8 \%$ $(15 / 16)]$ than in the group II $[56.3 \%(9 / 16)], \mathrm{p}=0.0158$.

\section{Discussion}

The results of this study showed the efficacy of OnabotA administration for the treatment of adult patients with upper and/or lower limb PSS, according to the improvement in the MAS score.

Patients receiving OnabotA at early stages of the process had a greater decrease in LL MAS score and need to be infiltrated in a lower number of muscles than those treated in more advanced phases. Moreover, these patients treated in early stages, required a lower dose of OnabotA at baseline which decreased throughout the study follow-up. Conversely, patients treated in late phases required higher doses of OnabotA that increased throughout the study, probably due to a worse evolution of the pathology.

Onabotulinumtoxin A has been proposed as a part of effective integrated treatment program for the management of PSS [32].
The results of 5E study [10] have shown that, in Spain, the $79 \%$ of patients with spasticity received treatment with BoNTA. Furthermore, the scientific evidence supported a level a recommendation for BoNTA for the spasticity treatment, both in the upper and lower limb [33].

Until few years ago, treatment of spasticity was usually delayed until secondary complications are established, approximately 6 months after the stroke [20]. However, there is scientific evidence suggesting that delay the treatment of spasticity, until secondary complications are established implies the irreversibility of the process and condemns to a local or systemic lifetime treatment for spasticity $[26,34]$.

The efficacy and safety of BoNTA for the treatment of spasticity in clinical practice has been widely demonstrated [15-21]. However, so far, the advantages of establishing treatment at early stages of the process have not been fully demonstrated.

It has been suggested that treatment of spasticity should be started early in the process, not only with physical therapy [35] but also with BoNTA [36,37]. Hesse et al., in a prospective study evaluated whether an early BoNTA injection, in subacute stroke patients, may prevent a disabling finger flexor stiffness six months later [36]. The results of this study found a positive effect of the BoNTA injection on finger flexor stiffness, presumably attributable to a reduced contracture development [36].

Cousin et al., in a double-blind randomized placebo-controlled trial evaluated whether a low dose of BoNTA, given early post stroke before clinically evident spasticity warrants treatment, will improve recovery of arm function [37]. The results of this study showed that low doses of BoNTA given at early stages of the process improved the recovery of arm function [37].

Moreover, the results of a meta-analysis, which aimed to evaluate the effects of BoNTA injection at early stages of PSS, demonstrated the beneficial effects of BoNTA treatment on improving hypertonicity within 3 months post-stroke [23].

This study was focused in mimic the real clinical practice conditions, that is why we selected, as cut-off point, the 6 months after stroke. Additionally, interventions were performed according to the needs of the patient, which reflects more accurately the reality of spasticity.

Moreover, it is noteworthy that total dose of OnabotA administered throughout the study follow-up was significantly lower in the patients who initiated treatment of spasticity earlier. This finding seems to indicate that early BoNTA administration may modify the course of the disease and reduce the incidence of PSS [23,36,37].

The results of this study are similar to those afore mentioned. A greater reduction in Ashworth scores was observed in patients receiving OnabotA treatment at early stages and, in addition, the OnabotA dose administered throughout the study was significantly lower in patients who started treatment with OnabotA before.

Therefore, the significant correlation observed between the time elapsed between the stroke onset and the start of OnabotA treatment and the final values of MAS, in both UL and LL; pain assessment; number of LL infiltrated muscles and OnabotA dose administered 
Citation: Vázquez A, Caballero JA, Spottorno MP, García E, García L, et al. (2018) Efficacy of Early Treatment with Onabotulinum Toxin A in Post-Stroke Spasticity. J Phys Med Rehabil Disabil 4: 025.

- Page 6 of $7 \bullet$

throughout the study follow-up supports the fact that the initiation of OnabotA treatment at early stages of the PSS process was associated with a better evolution of the condition.

The results of a phase II clinical trial evaluating BoNTA treatment associated with early-stage physiotherapy in patients with upper limb spasticity following stroke [4] are pending. As far as we know, the design and the initial values have been published, but the results are not known.

The dosages and administration techniques used in our study are in line with the recommendations of the guidelines and consensus $[38,39]$, although the total mean dose administered at baseline $(365$ $\mathrm{U})$ was slightly higher than that published by the $5 \mathrm{E}$ study $(278 \mathrm{U})$ [10].

There are limitations of our study that need to be mentioned. Selection bias, observational bias, and confounding are all inherent limitations of retrospective studies. Nevertheless, the strict inclusion/ exclusion criteria applied in our study were such to minimize this potential bias. The study design does not allow us to make decisive conclusions about the early OnabotA administration. The second limitation is the single-center nature of the study, with a limited number of patients. Nevertheless, the sample size was calculated prior the study. Another important limitation of this study is the use of only one scale for assessing spasticity. The change in MAS have been used in different studies $[40,41]$. Although there are certain issues concerning validity and reliability, Ashworth Scales are the most widely used tests to assess the severity of muscle spasticity [42]. In fact, recently published studies have shown that inter- and intra-rater agreement for Modified Ashworth Scale scores was satisfactory $[43,44]$.

In order to minimize this fact, the study assessed other different parameters, such as functional capacity by using a generic scale such as Barthel; pain assessment by means a VAS; the evaluation of needs of concomitant treatment (which compensates for the subjectivity of the VAS); total dose of infiltrated BoNTA; and number of infiltrated muscles.

Additionally, it is important to have into consideration that this is focused in mimic the real clinical practice conditions and the Healthcare pressure does not allow, in many cases, perform many different spasticity scales.

An additional limitation may be the difference in the concomitant medication regimes between the two groups. Nevertheless, this finding may be explained by the fact that those patients that started the treatment at early stages required less concomitant treatments.

Despite these limitations, the results of this study suggested that OnabotA treatment administered at early stages in post-stroke spasticity patients was associated with a better evolution of the process. Further studies are needed, especially to elucidate whether OnabotA administered at early stages after stroke might modify the course of the disease.

\section{Acknowledgement}

Editorial assistance in the preparation of this manuscript was provided by Antonio Martinez (MD) of Ciencia y Deporte S.L. Support for this assistance was funded by Allergan S.A.

\section{Funding}

Support for Medical writing assistance was funded by Allergan S.A.

\section{Conflict of Interest}

No potential conflict of interest was reported by the authors.

\section{References}

1. Martin A, Abogunrin S, Kurth H, Dinet J (2014) Epidemiological, humanistic, and economic burden of illness of lower limb spasticity in adults: A systematic review. Neuropsychiatr Dis Treat 10: 111-122.

2. Gillard PJ, Sucharew H, Kleindorfer D, Belagaje S, Varon S, et al. (2015) The negative impact of spasticity on the health-related quality of life of stroke survivors: A longitudinal cohort study. Health Qual Life Outcomes 13: 159 .

3. Pandyan AD, Gregoric M, Barnes MP, Wood D, Van Wijck F, et al. (2005) Spasticity: Clinical perceptions, neurological realities and meaningful measurement. Disabil Rehabil 27: 2-6.

4. Lindsay C, Simpson J, Ispoglou S, Sturman SG, Pandyan AD (2014) The early use of botulinum toxin in post-stroke spasticity: Study protocol for a randomised controlled trial. Trials 15: 12

5. Wissel J, Verrier M, Simpson DM, Charles D, Guinto P, et al. (2015) Poststroke spasticity: Predictors of early development and considerations for therapeutic intervention. PM R 7: 60-67.

6. Turner-Stokes L, Fheodoroff K, Jacinto J, Maisonobe P, Zakine B (2013) Upper limb international spasticity study: Rationale and protocol for a large, international, multicentre prospective cohort study investigating management and goal attainment following treatment with botulinum toxin A in real-life clinical practice. BMJ Open 3.

7. Ryu JS, Lee JW, Lee SI, Chun MH (2010) Factors predictive of spasticity and their effects on motor recovery and functional outcomes in stroke patients. Top Stroke Rehabil 17: 380-388.

8. Lundström E, Terént A, Borg J (2008) Prevalence of disabling spasticity 1 year after first-ever stroke. Eur J Neurol 15: 533-539.

9. Welmer AK, Holmqvist L Widen, Sommerfeld DK (2010) Location and severity of spasticity in the first 1-2 weeks and at 3 and 18 months after stroke. Eur J Neurol 17: 720-725.

10. Garreta-Figuera R, Torrequebrada-Giménez A, en representación del Grupo de Estudio 5E [2016]. Evaluación del tratamiento de la espasticidad en España: estudio 5E. Rev Neurol 63: 289-296.

11. Sommerfeld DK, Eek EU, Svensson AK, Holmqvist LW, Von Arbin MH (2004) Spasticity after stroke: Its occurrence and association with motor impairments and activity limitations. Stroke 35: 134-139.

12. Watkins CL, Leathley MJ, Gregson JM, Moore AP, Smith TL, et al. (2002) Prevalence of spasticity post stroke. Clin Rehabil 16: 515-22.

13. Bergfeldt U, Borg K, Kullander K, Julin P (2006) Focal spasticity therapy with botulinum toxin: Effects on function, activities of daily living and pain in 100 adult patients. J Rehabil Med 38: 166-171.

14. Karri J, Mas MF, Francisco GE, Li S (2017) Practice patterns for spasticity management with phenol neurolysis. J Rehabil Med 49: 482-488.

15. Royal College of Physicians (2009) Spasticity in adults: Management using Botulinum Toxin. National guidelines. Royal College of Physicians, England, UK.

16. Sheean G, Lannin NA, Turner-Stokes L, Rawicki B, Snow BJ, et al. (2010) Botulinum toxin assessment, intervention and after-care for upper limb hypertonicity in adults: International consensus statement. Eur J Neurol 17: 74-93. 
Citation: Vázquez A, Caballero JA, Spottorno MP, García E, García L, et al. (2018) Efficacy of Early Treatment with Onabotulinum Toxin A in Post-Stroke Spasticity. J Phys Med Rehabil Disabil 4: 025.

17. Santamato A, Micello MF, Ranieri M, Valeno G, Albano A, et al. (2015) Employment of higher doses of botulinum toxin type A to reduce spasticity after stroke. J Neurol Sci 350: 1-6.

18. Fheodoroff K, Ashford S, Jacinto J, Maisonobe P, Balcaitiene J, et al. (2015) Factors influencing goal attainment in patients with post-stroke upper limb spasticity following treatment with botulinum toxin A in real-life clinical practice: Sub-analyses from the Upper Limb International Spasticity (ULIS)-II study. Toxins (Basel) 7: 1192-1205.

19. Gastaldi L, Lisco G, Pastorelli S, Dimanico U (2015) Effects of botulinum neurotoxin on spatio-temporal gait parameters of patients with chronic stroke: A prospective open-label study. Eur J Phys Rehabil Med 51: 609 618.

20. Dong Y, Wu T, Hu X, Wang T (2017) Efficacy and safety of botulinum toxin type A for upper limb spasticity after stroke or traumatic brain injury: A systematic review with meta-analysis and trial sequential analysis. Eur J Phys Rehabil Med 53: 256-267.

21. Saita K, Morishita T, Hyakutake K, Fukuda H, Shiota E, et al. (2017) Combined therapy using botulinum toxin A and single-joint hybrid assistive limb for upper-limb disability due to spastic hemiplegia. J Neurol Sci 373: 182-187.

22. López de Munaín L, Juan-García FJ, Duarte E, Martín-Mourelle R, Rodríguez S, et al. (2016) Tratamiento farmacológico temprano de la espasticidad postictus con toxina botulínica tipo A: Recomendaciones de consenso basadas en la evidencia. Rev Neurol 63: 363-369.

23. Rosales RL, Efendy F, Teleg ES, Delos Santos MM, Rosales MC, et al (2016) Botulinum toxin as early intervention for spasticity after stroke or non-progressive brain lesion: A meta-analysis. J Neurol Sci 371: 6-14.

24. Lieber RL, Steinman S, Barash IA, Chambers H (2004) Structural and functional changes in spastic skeletal muscle. Muscle Nerve 29: 615-627.

25. NIH Stroke Scale, National Stroke Association, Colorado, USA.

26. Bohannon RW, Smith MB (1987) Interrater reliability of a modified ashworth scale of muscle spasticity. Phys Ther 67: 206-207.

27. Ansari NN, Naghdi S, Moammeri H, Jalaie S (2006) Ashworth scales are unreliable for the assessment of muscle spasticity. Physiother Theory Pract 22: $119-125$.

28. Wissel J, Schelosky LD, Scott J, Christe W, Faiss JH, et al. (2010) Early development of spasticity following stroke: A prospective, observational trial. J Neurol 257: 1067-1072.

29. Olver J, Esquenazi A, Fung VSC, Singer BJ, Ward AB (2010) Botulinum toxin assessment, intervention and aftercare for lower limb disorders of movement and muscle tone in adults: International consensus statement. Eur J Neurol 2: 57-73.

30. Haefeli M, Elfering A (2006) Pain assessment. Eur Spine J 1: 17-24.
31. Cid-Ruzafa J, Damián-Moreno J (1997) [Disability evaluation: Barthel's index]. Rev Esp Salud Publica 71: 127-137.

32. Orsini M, Leite MA, Chung TM, Bocca W, de Souza JA, et al. (2015) Botulinum neurotoxin type a in neurology: Update. Neurol Int 7: 5886.

33. Esquenazi A, Albanese A, Chancellor MB, Elovic E, Segal KR, et al (2013) Evidence-based review and assessment of botulinum neurotoxin for the treatment of adult spasticity in the upper motor neuron syndrome. Toxicon 67: 115-128.

34. Opheim A, Danielsson A, Alt Murphy M, Persson HC, Sunnerhagen KS (2015) Early prediction of long-term upper limb spasticity after stroke: Part of the SALGOT study. Neurology 85: 873-880.

35. Langhorne P, Stott D, Knight A, Bernhardt J, Barer D, et al. (2010) Very early rehabilitation or intensive telemetry after stroke: A pilot randomised trial. Cerebrovasc Dis 29: 352-360.

36. Hesse S, Mach H, Fröhlich S, Behrend S, Werner C, et al. (2012) An early botulinum toxin A treatment in subacute stroke patients may prevent a disabling finger flexor stiffness six months later: A randomized controlled trial. Clin Rehabil 26: 237-245.

37. Cousins E, Ward A, Roffe C, Rimington L, Pandyan A (2010) Does lowdose botulinum toxin help the recovery of arm function when given early after stroke? A phase II randomized controlled pilot study to estimate effect size. Clin Rehabil 24: 501-513.

38. Simpson DM, Patel AT, Alfaro A, Ayyoub Z, Charles D, et al. (2017) OnabotulinumtoxinA injection for poststroke upper-limb spasticity: Guidance for early injectors from a delphi panel process. PM R 9: 136-148.

39. Esquenazi A, Alfaro A, Ayyoub Z, Charles D, Dashtipour K, et al. (2017) OnabotulinumtoxinA for lower-limb spasticity: Guidance from a delphi panel approach. PM R 9: 960-968.

40. Guarany FC, Picon PD, Guarany NR, dos Santos AC, Chiella BP, et al. (2013) A double-blind, randomised, crossover trial of two botulinum toxin type a in patients with spasticity. PLoS One 8: 56479.

41. Do KH, Chun MH, Paik NJ, Park YG, Lee SH, et al. (2017) Safety and efficacy of letibotulinumtoxinA (BOTULAX $\left.{ }^{\circledR}\right)$ in treatment of post stroke upper limb spasticity: A randomized, double blind, multi-center, phase III clinical trial. Clinical Rehabilitation 31: 1179-1188.

42. Ansari NN, Naghdi S, Moammeri H, Jalaie S (2006) Ashworth scales are unreliable for the assessment of muscle spasticity. Physiother Theory Pract 22: $119-125$.

43. Li F, Wu Y, Li X (2014) Test-retest reliability and inter-rater reliability of the modified tardieu scale and the modified ashworth scale in hemiplegic patients with stroke. Eur J Phys Rehabil Med 50: 9-15.

44. Meseguer-Henarejos AB, Sánchez-Meca J, López-Pina JA, Carles-Hernández R (2017) Inter-and intra-rater reliability of the modified ashworth scale: A systematic review and meta-analysis. Eur J Phys Rehabil Med. 